\title{
REFUGIADOS CLIMÁTICOS: ¿QUIÉN DEBE CARGAR LOS COSTOS?
}

\author{
Daniel Loewe
}

\begin{abstract}
El artículo ${ }^{1}$ argumenta a favor del concepto de "refugiado climático" y sostiene que la responsabilidad por su desplazamiento así como los costos asociados, incluyendo su recepción en otros Estados, deben ser asignados entre los Estados en razón de su uso del recurso atmosférico así como del beneficio que ese uso les reporta.
\end{abstract}

Palabras claves: cambio climático, desplazamiento forzado, refugiado climático, principios de asignación de costos.

La evidencia de los cambios medioambientales en las últimas décadas ha dirigido la atención al tema de los refugiados medioambientales: individuos que deben abandonar su lugar de origen en razón de la degradación del medio ambiente que torna inviable o difícil la vida humana.

Las causas de esta degradación son múltiples. Hay desplazamientos retrotraibles a la acción humana directa (como, por ejemplo, la construcción de represas). Otros que se retrotraen a eventos de la naturaleza que no guardan relación con la acción humana (erupciones volcánicas, terremotos, etc.). Y otros desplazamientos que se tienden a asociar con la acción humana indirecta. Entre estos últimos se cuentan especialmente los que se relacionan con el calentamiento global.

También son diversos los tipos de desplazamiento que se relacionan con la degradación medioambiental. La gran mayoría de los desplazados lo son dentro de las fronteras políticas de su Estado de origen. Pero otros deben abandonar sus países. Algunos desplazados lo son en forma temporal, hasta que las condiciones medioambientales tornen nuevamente habitable la geografía.

\footnotetext{
* Universidad Adolfo Ibáñez, Escuela de Gobierno, Centro de Investigación en Teoría Política y Social. Santiago/Chile.

1 Este artículo forma parte del Proyecto de Investigación Fondecyt (1120736).
} 
Otros desplazamientos son vías sin retorno. Este es el caso de los ya mencionados desplazados por la construcción de represas. Pero también, y central para este artículo, es el caso de muchos desplazados medioambientales que se relacionan con el calentamiento global. Un caso ejemplar es el de algunos Estados-islas.

En este artículo me referiré específicamente al caso del desplazamiento humano forzado por razones medioambientales producto del cambio climático que va de la mano del calentamiento global. Al referirme a este tipo de casos utilizaré el concepto de "refugiado climático". Tanto el tema del cambio climático como el de la migración y los refugiados están en el centro de la atención no sólo académica y política, sino también de la opinión pública. Pero los desarrollos filosóficos con respecto a este fenómeno son incipientes y moderados.

A continuación investigo de un modo esquemático la asignación de responsabilidad por los refugiados climáticos y así de los costos asociados. En primer lugar me refiero al fenómeno del desplazamiento humano en razón del calentamiento global y al concepto de refugiado climático. Luego me refiero a los costos del desplazamiento climático desde una perspectiva normativa de justicia igualitaria. En tercer lugar me refiero a tres principios de asignación de costos asociados con los refugiados climáticos y argumento a favor de un principio compuesto. Finalmente examino concisamente algunas preguntas relativas a la aplicación de este principio.

\section{Refugiados climáticos}

Ya en 1990 el Intergovernmental Panel on Climate Change (IPCC) notó que el mayor impacto singular del cambio climático sería en la migración humana. Los flujos migratorios se relacionarían con la inundación y erosión de las costas, desertificación y destrucción de la agricultura, variación en los regímenes de Iluvias, mayor prevalencia de fenómenos naturales que afectan negativamente la vida humana, como tornados y olas de calor, etc.

El tema del desplazamiento forzado por el cambio climático no es sólo importante por sus consecuencias en el bienestar de los individuos. Además, en razón de consideraciones geopolíticas, es urgente. Por cierto, es difícil establecer la magnitud de los desplazamientos y así de las consideraciones geopolíticas y las consecuencias en el bienestar agregado. Ellos dependen del escenario de cambio climático. Y estos van desde cambios tenues a cambios substanciales (los 2 grados de calentamiento global usualmente aceptados como objetivo mundial) e incluso dramáticos (más de 4 o 5 grados). También depende de las políticas demográficas de crecimiento en los diversos países. Pero además depende estrechamente de las políticas de mitigación y adaptación que se realicen en los diferentes países, y esto último también se relaciona con el desarrollo económico. 
Los escenarios modulados varían entre 25 y 1000 millones de desplazados medioambientales en el año 2050. A pesar de la imposibilidad de establecer predicciones con pretensión de exactitud, la cifra presentada por $\mathrm{Myers}^{2}$ de 200 millones de desplazados medioambientales ese año ha llegado a ser ampliamente aceptada, siendo recogida en influyentes estudios como el Stern Review sobre la economía del cambio climático ${ }^{3}$ y en las publicaciones del IPCC. Por cierto, estas cifras son también criticadas en razón de su carácter especulativo y alarmista ${ }^{4}$. Si el escenario de 200 millones fuese efectivo, esto significaría que de acuerdo a las predicciones de crecimiento demográfico, el año 2050 uno de cada 45 individuos en el mundo sería un desplazado medioambiental.

Aunque los modelos son complejos e imprecisos, se acepta como un hecho de la causa que la cantidad de desplazados medioambientales aumentará en el futuro. Objeto de discusión es el tipo de relación que se establece entre la migración humana y la degradación medioambiental producto del cambio climático. En ocasiones se establece una relación causal directa entre el cambio climático y la migración. Es el caso ejemplar de los habitantes de los Estados Islas del Pacífico ${ }^{5}$. En estos casos parece establecerse una relación causal directa entre el cambio climático y el desplazamiento humano. Kiribati, por ejemplo, es un Estado compuesto de islas que en algunos casos apenas se elevan por sobre el nivel del mar. Además de las inclemencias del tiempo, está amenazada por el aumento del nivel del mar que, aunque no haga desaparecer todas las islas, saliniza el agua y la tierra, tornándolas en inhabitables. Parte del problema se relaciona con la gran población de las islas, que se estima en más de cien mil personas. Y los líderes de Tuvalu (una pequeña isla en el océano pacífico, entre Hawái y Australia) ya han anunciado que abandonarán su isla ${ }^{6}$.

En otras ocasiones se considera el cambio climático como un elemento más entre otros, y no necesariamente el más relevante, en la ecuación explicativa de los flujos: "Migrants respond to economic, social, and demographic factors in addition to the environment. Assessing environmental influences on migration is complex and must take these other factors into account" ${ }^{\prime \prime}$. Ciertamente el riesgo que implica el cambio climático para el bienestar humano está mediado por la capacidad y disposición para hacer frente a esos riesgos. Como es conocido,

2 MYERS, Norman. Environmental Refugees: An emergent security issue.

3 STERN, Nicholas (ed.). The economics of climate change.

4 Compare BLACK, Richard. Environmental refugees: myth or reality?

5 MINURA, Nobuo et alii. Small Island; MORTREUX, Colette e BARNETT, Jon. Climate change, migration and adaptation in Funafuti, Tuvalu.

6 BROWN, Lester R. Environmental refugees.

7 Compare GRAY, Clark. Environmental refugees or economic migrants?, p. 6, para múltiples referencias a literatura empírica contraria a la idea de la relación directa entre degradación medioambiental y grandes flujos migratorios. 
los riesgos y las consecuencias negativas del cambio climático no se distribuyen equitativamente. Usualmente son los más pobres los que deberán cargar con las consecuencias más negativas porque disponen - por causas diversas, entre las que se cuenta la falta de recursos, pero también la administración ineficiente de menos oportunidades para implementar medidas de mitigación y adaptación. Así, en ocasiones, el cambio climático es uno más entre otros factores a la base del desplazamiento humano. Por ejemplo, una parte de los denominados inmigrantes económicos buscan horizontes más prometedores en razón de, entre otros, los efectos de la degradación medioambiental en la economía de sus zonas de origen. De cualquier modo, es evidente que el cambio climático con los riesgos para el bienestar humano que conlleva es al menos parcialmente causante del desplazamiento involuntario. $\mathrm{Y}$ esto es relevante para cualquier asignación de responsabilidad por los refugiados climáticos.

A pesar de la urgencia geopolítica y las consecuencias negativas en el bienestar humano, hay reticencia en la consideración de los desplazados medioambientales como refugiados climáticos. Aunque este concepto comienza a ser utilizado en las ciencias sociales, es rechazado por el derecho internacional ${ }^{8}$. Así ha quedado recientemente establecido por una Corte de Justicia neozelandesa que rechazó la demanda del ciudadano de Kiribati, loane Teitiotas, para ser reconocido como el primer refugiado climático ${ }^{9}$.

El derecho internacional respalda la decisión de la Corte neozelandesa. Bajo la presión de los movimientos masivos de refugiados a finales de la segunda guerra mundial surgieron los instrumentos jurídicos que, con algunas modificaciones, otorgan la base al derecho de asilo contemporáneo. El instrumento internacional más importante es la Convención de Refugiados de Ginebra (CRG) del 28 de julio de 1951. En primer lugar, se limitó a las olas de refugiados que siguieron al mencionado conflicto bélico. Sin embargo, mediante un protocolo añadido en 1967 adquirió validez universal. Importante en la CRG es la definición de refugiado (art. 1, A2): toda persona que "debido a un temor fundado de persecución en razón de su raza, religión, nacionalidad, pertenencia a un grupo social particular o en razón de su convicción política, se encuentra

${ }_{8}$ La definición usual de "refugiado medioambiental" proviene de informe de El-Hinnawi del programa de medioambiente de las Naciones Unidas: "Environmental refugees are defined as those people who have been forced to leave their traditional habitat, temporary or permanently, because of a marked environmental disruption (natural and/or triggered by people) that jeopardized their existence and/or seriously affected the quality of their life. By 'environmental disruption' in this definition is meant any physical, chemical and/or biological changes in the ecosystem (or the resource base) that render it, temporarily or permanently, unsuitable to support human life" (ELHINNAWI. Environmental Refugees, p. 4).

9 Cf. <http://www.independent.co.uk/news/world/australasia/worlds-first-climate-change-refugeehas-appeal-rejected-as-new-zealand-rules-ioane-teitiota-must-return-to-south-pacific-islandnation-of-kiribati-9358547.html>. 
fuera del país cuya nacionalidad posee, y que no puede demandar la protección de éste o en razón de esos temores fundados no la quiere demandar" debe ser considerada como refugiado. El núcleo duro de la CRG lo constituye el artículo 33.1, que establece la prohibición de expulsión - que debido a la versión original francesa ha encontrado cabida en el derecho internacional como la condición de non-refoulement. De acuerdo a esta condición:

ninguno de los estados partes de este contrato expulsará de algún modo a un refugiado sobre las fronteras de los territorios en los que su vida o su libertad, en razón de su raza, religión, ciudadanía, su pertenencia a un grupo social particular o en razón de su convicción política, fuese amenazada.

Ciertamente, aquí no hay espacio para la categoría de refugiado climático. Por una parte, la categoría referida a la adversidad climática o degradación medioambiental no se encuentra disponible. Por otra parte, la devastación medioambiental producto del cambio climático no es persecución, aunque las consecuencias puedan ser igualmente negativas en los dos casos. Y según las Naciones Unidas, la situación de aquellos que no son reconocidos legalmente como refugiados es similar a la de los extranjeros ilegales y pueden, por tanto, ser expulsados - lo que presumiblemente sucederá con loane Teitiotas. Grupos de derechos humanos y representantes de Estados-islas luchan desde hace años por el reconocimiento de los refugiados climáticos. Pero lo único que han logrado es que en la cumbre de Cancún, los participantes acordaran comprensión y trabajo en conjunto en relación a la expulsión, migración y desplazamiento en razón de clima. Hasta ahora, esa comprensión y trabajo en conjunto no ha significado más que una declaración de buenas intenciones sin consecuencias prácticas.

Es usual criticar el concepto de refugiado medioambiental o climático por ser "unhelpful and unsound intellectually, and unnecessary in practical terms"10. Pero las argumentaciones en que se basa la crítica a la extensión conceptual propuesta son poco plausibles. Las críticas refieren a la falta de evidencia empírica del fenómeno del desplazamiento medioambiental; a la simplificación que implica considerar que estos desplazamientos son por causas "medioambientales"; y a que es un error estratégico referir a los desplazados medioambientales como "refugiados medioambientales", porque así se los asimila a los "refugiados económicos" reduciendo, por tanto, la responsabilidad de los Estados receptores con respecto a ellos al tratarlos de un modo análogo a estos últimos ${ }^{11}$.

Primero, la evidencia empírica es objeto de debate. Pero incluso considerando que las cifras de desplazados medioambientales hoy y sobre todo

10 BLACK, op. cit.

11 Para estas críticas y su discusión, compare BELL, Derek R. Environmental refugees: what rights? which duties? 
a futuro son especulativas, las discusiones en torno a ellas pueden interpretarse como una razón para ahondar las investigaciones al respecto, o para restringir el rango de efectos que trae el desplazamiento medioambiental en caso que se concluya que los flujos serán menores. Pero ciertamente no se pueden interpretar como una razón para considerar que los desplazados medioambientales no son refugiados. Afirmar lo contrario se retrotrae a un error categorial. Segundo, el que las causas explicativas del desplazamiento sean múltiples, no implica que el cambio climático no sea, aunque parcialmente, una de ellas. Y tercero, no es ni evidente ni convincente que la responsabilidad de los Estados receptores por los refugiados medioambientales se reduzca al seguir la analogía con los refugiados económicos - como no es evidente ni convincente que los refugiados económicos deban ser considerados una subclase menos privilegiada dentro de los refugiados. A la base de la crítica señalada está probablemente la pretensión de reservar el concepto de refugiado exclusivamente para los casos de persecución estipulados en la CRG. Pero esta pretensión no es convincente, al menos no, si aceptamos algún principio cosmopolita, aunque sea moderado, que regle normativamente las relaciones entre individuos y Estados ajenos. De la aceptación de un principio cosmopolita se sigue el reconocimiento de un derecho de refugio toda vez que las condiciones fácticas hagan imposible la vida o ciertas condiciones de vida consideradas como mínimamente aceptables en razón de alguna concepción normativa. Y este derecho es al menos efectivo en tanto los Estados de origen no se puedan hacer cargo o no estén dispuestos a hacerse cargo del mejoramiento de las condiciones fácticas de la vida.

Esto se puede deducir, por ejemplo, de un derecho cosmopolita minimalista como el defendido por Kant en Sobre la Paz Perpetua ${ }^{12}$. La idea del derecho cosmopolita kantiano se basa en que la corporalidad del hombre implica un derecho original a poder estar en algún lugar sobre la superficie de la tierra ${ }^{13}$. Pero ya que la tierra es esférica y por tanto su superficie limitada, los hombres no se pueden dispersar infinitamente. Por tanto, el límite del suelo obliga a un principio según el cual los hombres sólo pueden hacer uso de un sitio sobre la tierra siguiendo leyes de derecho. El derecho cosmopolita regla las relaciones entre individuos y Estados ajenos. De este modo, por ejemplo, aquel que a causa de un naufragio es arrojado a la costa de un Estado al que no pertenece, tiene un derecho de visita, porque por su corporalidad precisa de un suelo bajo sus pies. El derecho cosmopolita kantiano es muy limitado en sus exigencias normativas. Kant lo entiende exclusivamente como un derecho de hospitalidad pero no de permanencia. Por cierto a Kant le interesaba especialmente posibilitar las

\footnotetext{
${ }^{12}$ Este argumento en LOEWE, Daniel. Los náufragos de nuestro mundo. El caso de los refugiados.

${ }^{13}$ KANT, Immanuel. La Metafísica de las Costumbres; IDEM. Hacia la Paz Perpetua.
} 
relaciones comerciales, así como imposibilitar el colonialismo, al que se oponía. El derecho cosmopolita, entendido como un derecho de hospitalidad, hace posible lo primero e imposible lo segundo. Sin embargo, a pesar de su minimalismo normativo, el derecho cosmopolita kantiano implica que toda vez que el hábitat natural haga imposible las condiciones fácticas de la vida, se dispone de un derecho de refugio, aunque sea temporalmente limitado. Así, los desplazados climáticos deben ser considerados como refugiados climáticos, toda vez que las condiciones fácticas hagan imposible la vida humana, o una vida humana por sobre algún nivel mínimo de calidad estipulado de un modo normativo.

\section{Igualitarismo y los costos del desplazamiento climático}

La relación entre clima y migración no es nueva. Ella ha estado en el centro del desarrollo de la humanidad. Lo que la torna en objeto de la atención de la filosofía política y moral son consideraciones de justicia. Un modo de introducir la pregunta acerca de la justicia en relación al desplazamiento producto del cambio climático es examinar la asignación de responsabilidad. ¿Qué entidades son responsables de los desplazamientos involuntarios producto del cambio climático? Si asumimos que (a) el cambio climático tiene causas antropogénicas importantes, (b) y notamos que tiene impactos negativos en la vida humana tales como los desplazamientos involuntarios, (c) y que mitigar o hacerse cargo de este impacto implica costos, entonces, recurriendo a las palabras de Henry Shue, resulta fundamental plantearse la pregunta sobre "the justice of the international allocation of the cost of dealing with global environmental problems"114. En este caso, la pregunta sobre la justicia de la asignación internacional de los costos relativos a las políticas migratorias en razón del cambio climático.

En la literatura se suelen reconocer dos tipos de costos. Por una parte están los costos de mitigación, que surgen debido a políticas que apuntan a eliminar, disminuir o acotar las actividades humanas que aumentan el calentamiento global generando un impacto negativo en la vida humana y/o animal. Este tipo de costos surge, por ejemplo, al reducir las emisiones de gases de efecto invernadero.

Por otra parte están los costos de adaptación, que surgen en razón de políticas que apuntan a facilitar y apoyar la adaptación a los cambios climáticos. Entre estos se cuentan, por ejemplo, la construcción de diques para contener el nivel del mar en zonas bajas, la inoculación contra enfermedades infecciosas, ayuda a los empobrecidos debido al cambio climático, irrigación de zonas desertificadas, etc. Pero también, y relevante para el tema de este artículo, subvencionar la adaptación de desplazados medioambientales a nuevos contextos geográficos, económicos y sociales.

\footnotetext{
${ }^{14}$ SHUE, Henry. The Unavoidability of Justice, p. 373.
} 
Sin embargo, más allá de los mencionados, en el caso de los refugiados climáticos hay que hacerse cargo de la asignación de otros dos tipos de costos que la literatura sobre la justicia y el cambio climático no considera. Por una parte está el costo directo para los Estados que se deriva de una obligación de aceptar refugiados climáticos. Estos son los costos de aceptación. Por otra parte, aunque se restrinjan las prácticas que producen calentamiento global y se facilite la adaptación a las nuevas condiciones climáticas, asignándose de algún modo los costos de mitigación y adaptación, es razonable suponer que muchos individuos estarán peor de lo que estarían si no hubiesen sufrido los efectos negativos del cambio climático. Si este es el caso, habría también costos de reparación e indemnización. Por ejemplo, no sólo debe ser facilitada la adaptación de los desplazados medioambientales a nuevos contextos geográficos, económicos y sociales. Ya que estos desplazados habrán perdido aspectos importantes y valiosos, como la vida comunal, prácticas ancestrales, vínculos con el paisaje y el pasado, etc. ${ }^{15}$, sin ser los causantes de las condiciones a la base de su movilidad forzada, es razonable suponer que podrían reclamar algún tipo de indemnización.

¿Quién debe hacerse responsable por los costos asociados a los refugiados climáticos? Debido a las características de los daños medioambientales producto del cambio climático (acumulativos y por dispersión) y a la multiplicidad de actores implicados en su generación, la pregunta acerca de la asignación de responsabilidad es parte de la justicia global, en alguna de sus variantes ${ }^{16}$.

${ }^{15}$ Sobre la idea de la tierra como un hogar inalienable para aquellos que la habitan, compare SMITH, Mick. An ethics of place: radical ecology, postmodernity and social theory.

${ }^{16}$ Una característica singular de las consecuencias negativas para la vida humana que trae consigo el calentamiento global, tales como el desplazamiento forzado, es que tienen la forma de daños acumulativos. Es decir, el calentamiento global y sus daños no se pueden retrotraer a ningún agente en particular. Agente A produce gases contaminantes que, por si solos, no tienen efectos dañinos. Lo mismo hace agente $B$, y agente $C$, etc. Pero es la sumatoria de todos estos contaminantes, también en una perspectiva temporal, los que, a partir de algún momento, produce el daño. La situación es comparable a un atasco en la carretera. Suponiendo que la causa es la cantidad de usuarios, el daño no ha sido producido por ninguno de los automovilistas individualmente, sino que por el conjunto de todos ellos. Otra característica de los daños producto del calentamiento global es su dispersión. Esta dispersión puede tener una forma espacial o temporal. De acuerdo a la primera las acciones realizadas en una ubicación geográfica particular (en tanto parte de un proceso de generación de daños acumulativos) pueden ser dañinas en una ubicación completamente diferente. Es a lo que se refiere Peter Singer al afirmar que conducir automóviles sobremotorizados en países ricos produce inundaciones en Bangladesh (2004). De acuerdo a la dispersión temporal de los daños, ya que la capacidad del ecosistema para absorber algunos de los gases que producen el efecto invernadero es limitada y lenta, en muchos casos los efectos negativos del calentamiento global se producirán con posterioridad a la producción de los gases. De este modo la determinación de los agentes que deben llevar los costos debe referir necesariamente a las relaciones entre generaciones. No es casual que el tema de la justicia en relación al cambio climático haya sido especialmente considerado desde la perspectiva de autores preocupados por la justicia global (cf. GRUBB, Michael. Seeking fair weather: ethics and the international debate on climate change; SHUE, op. cit.; IDEM. Avoidable Necessity: Global Warming, International Fairness, and Alternative Energy; SINGER, Peter. One World: The Ethics of Globalization; CANEY, Simon. Cosmopolitan Justice, Responsibility, and Global Climate Change). 
Podemos partir desde un paradigma distributivo igualitario y luego guiarnos por un principio de justicia correctiva.

De acuerdo a un principio igualitario habría algún $X$ que debe ser distribuido de modo igualitario ${ }^{17}$. Según Amartya $\operatorname{Sen}^{18}$ no sería posible prescindir de la igualdad al articular un argumento de justicia. La justicia implica igualdad. La pregunta no es: ¿por qué igualdad?, sino: zigualdad de qué? La disputa refiere, entonces, a la métrica de la igualdad. Lo que caracterizaría a la justicia es un entendimiento relacional, como la igualdad del uno con el otro, según la métrica que se considere apropiada. Desde esta perspectiva podemos identificar lo que le corresponde a cada cual (de acuerdo a la métrica escogida). Pero es menos clara la determinación de los agentes responsables en la asignación de los costos asociados con garantizar a cada cual lo que le corresponde.

El principio correctivo es aquel Ilamado a restituir la justicia igualitaria cuando ha sido violada. Este principio complementa el principio igualitario en tanto, en razón de lo que a cada cual le corresponde, determina el modo de garantizar las demandas legítimas que surgen en caso que la justicia igualitaria haya sido violada.

¿Quién debe cargar los costos que implica la justicia correctiva? Hay múltiples posibilidades. Una posibilidad extrema es aceptar un principio

\footnotetext{
17 No me referiré a las posibles justificaciones de un principio distributivo igualitario ni a las consecuencias normativas que se siguen de él. Hay muchos tipos de argumentación. Por ejemplo, el así llamado "igualitarismo de la suerte" da una respuesta a la pregunta acerca de porqué la igualdad (definida según la distribución de algún conjunto de bienes - la así llamada "currency" de la justicia igualitaria) es relevante. Esta respuesta se basa en una intuición acerca del igual valor de los individuos en cuanto agentes morales. Hay muchos modos diferentes de entender el contraste entre suerte y agencia - como las diferencias entre "plain" y "option luck", circunstancias y decisiones, etc. - y por lo tanto hay muchos modos diferentes de trazar la frontera entre ellas (compare ARNESON, Richard. Luck egalitarianism and prioritarianism; COHEN, Gerald A. On the currency of egalitarian justice; DWORKIN, Ronald. Equality, luck, and hierarchy; TAN, Kok-Chor. Justice, institutions, and luck). Pero la preocupación con la igualdad o desigualdad en la distribución de algún tipo de bienes no pertenece exclusivamente a los igualitaristas de la suerte. Hay otras respuestas a la pregunta de porqué la igualdad es importante. El contraste al igualitarismo de la suerte está dado por el así llamado igualitarismo "social", "relacional" o "democrático" (compare SCHEFFLER, Samuel. What is egalitarianism?; IDEM. Equality and tradition; RAWLS, John. Justice as Fairness; FREEMAN, Samuel. Justice and the social contract). Desde esta perspectiva la justicia de un estado distributivo del mundo se debe juzgar desde la perspectiva de la calidad de las relaciones sociales-políticas relevantes (una interesante discusión de estas posiciones en SEGALL, Shlomi. Equality and opportunity, p. 16). Ciertamente, la igualdad es aquí central, y tiene importantes consecuencias. Pero la igualdad es importante porque las relaciones socio-políticas entre los miembros de un orden social o político son importantes, y no porque las personas como tales estén en una relación de igualdad moral fundada en la distinción entre suerte y agencia. En lo que sigue daré por sentado la corrección de algún patrón igualitario de distribución, sin referirme a su justificación. De cualquier modo, la argumentación desarrollada en este artículo en razón de los refugiados climáticos se ajusta mejor a la perspectiva del igualitarismo de la suerte interpretado de un modo global.

${ }^{18}$ SEN, Amartya. Equality of What.
} 
cosmopolita que implica la responsabilidad de todos. El otro extremo es asignar la responsabilidad exclusivamente a aquellos que violaron el principio igualitario. Esto último corresponde a nuestras intuiciones más básicas de la justicia: el que pone una causalidad en movimiento de un modo intencional o al menos consciente acerca de los riesgos involucrados, es responsable por los resultados.

El caso de los refugiados climáticos debemos articular una respuesta a la pregunta acerca del objeto de la justicia igualitaria y a la pregunta acerca de la asignación de responsabilidad en caso que la igualdad haya sido violada. En lo que sigue me referiré a la primera, mientras que la segunda pregunta será objeto de la próxima sección.

¿Distribución igualitaria de qué? Es posible responder a esta pregunta de modos diferentes. Hay muchas posibilidades. Entre otras, igualdad de dignidad, de bienestar, de acceso al bienestar, de recursos, de bienes primarios. Sen y Nussbaum proponen capacidades ${ }^{19}$. Yo propondré un entendimiento mucho más limitado que se funda en una concepción de derechos humanos básicos: recursos limitados no renovables (o con tasas de renovación muy lentas) cuyo agotamiento implica consecuencias negativas para la garantía de derechos humanos básicos, deben ser distribuidos prima facie de un modo igualitario, pero sólo hasta el punto en que esos derechos humanos básicos no sean violados. El desplazamiento forzado en razón del cambio climático implica la violación de derechos humanos básicos. Estos procesos están asociados con condiciones de vulnerabilidad extrema, de desaparición de modos de ganarse la vida y de modos de vida, separación de familias, surgimiento de conflictos que amenazan estos derechos, etc. Bajo el supuesto de que el cambio climático tiene causas antropogénicas que refieren a la emisión de gases de efecto invernadero, el recurso limitado no renovable, que en este caso debe ser distribuido de un modo igualitario hasta el punto explicitado, es el recurso atmosférico: todos los individuos ${ }^{20}$ deben ser considerados, prime

${ }^{19}$ En todo caso, el principio distributivo es suficientarista: asegurar un mínimo de capacidades necesarias para actuar con libertad efectiva (SEN, Amartya. Inequality Reexamined; IDEM. Development as Freedom), o para llevar poder desarrollar una vida con dignidad humana (NUSSBAUM, Martha C. Women and Human Development; IDEM. Frontiers of Justice).

${ }^{20}$ El concepto "todos" debe ser cualificado. Primero, lo entiendo de un modo individual: cada individuo. Así, concepciones utilitaristas que apuntan a maximizar el total o el promedio se oponen a esta concepción distributivista. Segundo: el concepto "todos" no refiere exclusivamente a los seres existentes, sino también a los potenciales de existencia previsible: en la medida en que podamos suponer que seguirán existiendo individuos sobre la tierra, cada individuo debe tener una porción igual al recurso atmosférico. Por cierto, este entendimiento se debe entender como idea regulativa. Esto se debe a que su operacionalización es difícil o imposible: como el número de generaciones y así de individuos es ilimitado (en tanto no sepamos fehacientemente o con razonable seguridad sobre el fin de la vida humana sobre la tierra en un punto temporal singular), debemos atender a algunas generaciones delante de nosotros y asegurar para todos aquellos individuos este derecho de un modo igualitario. 
facie, como portadores de una demanda igualmente legítima a utilizar el recurso atmosférico en igual cantidad ${ }^{21}$.

\section{Justicia correctiva y responsabilidad por los refugiados climáticos}

¿Cómo asignar la responsabilidad por los costos en caso de que los derechos de algunos a una igual parte de este recurso sean violados? Examinaré tres propuestas.

Una posibilidad es extender la responsabilidad a todos aquellos que pueden hacerse cargo de (los costos del) los refugiados climáticos. El principio subyacente es que la responsabilidad moral se extiende a todos aquellos que tienen la capacidad de hacerse cargo de la situación. Este principio es corriente en los debates acerca de la justicia global y la pobreza. Desde esta perspectiva se suele considerar que la obligación de mitigar la pobreza no es simplemente una obligación supererogatoria o de caridad, sino que es una obligación moral de carácter humanitario. De acuerdo a Peter Singer, si podemos evitar que se produzca algo moralmente malo sin sacrificar nada comparable, estamos obligados a hacerlo ${ }^{22}$. Por ejemplo, si podemos salvar a un niño desconocido ahogándose en una pileta (incluso sin ser responsables de su situación) al costo de estropear nuestros zapatos, estamos moralmente obligados a hacerlo. Por paridad de razonamiento, si podemos aliviar la pobreza (la pobreza que mata, como señala Singer) mediante donaciones, estamos moralmente obligados a hacerlo, independientemente de la ubicación geográfica o de la nacionalidad de los afectados. Extendiendo la aplicabilidad al caso de los desplazados medioambientales: si podemos asumir los costos (de mitigación, adaptación, aceptación y compensación) estamos obligados a hacerlo. Este principio tiene cierta plausibilidad. Sin embargo, debido a su carácter normativo indeterminado, no lo consideraré como primeramente válido en el caso de la asignación de costos por los refugiados climáticos. En el mejor de los casos, tiene un rol subsidiario.

La segunda respuesta es que todos aquellos que utilizaron más que su parte deben hacerse cargo de los costos relacionados a su sobreconsumo. Esta idea se puede relacionar - sin ser lo mismo - con el muy discutido principio:

${ }^{21}$ Evidentemente esta propuesta se opone a interpretaciones lockianas, de acuerdo a las cuales cada cual puede apropiarse de todo aquello con lo que mezcle su trabajo, sacándolo así del estado de naturaleza. Pero aun en este caso, la interpretación lockiana debe cumplir con sus dos provisos: que quede suficiente y tan buenos para los otros; y que lo apropiado no se estropee. Y si interpretamos "que quede suficiente" de tal modo que implique que todos los demás puedan seguir apropiándose del recurso sin que sus derechos fundamentales sean violados (y esta es la misma lógica de Locke, sólo que la referencia es a derechos naturales de origen divino), entonces es posible incluso desde esta perspectiva derivar algún principio normativo acerca de la distribución del recurso atmosférico. En todo caso, no se trata necesariamente de un principio igualitario.

${ }^{22}$ SINGER, Peter. Famine, Affluence, and Morality; IDEM. The Life You Can Save. 
el que contamina paga. Este principio se suele sostener en las discusiones internacionales y en los documentos directrices que establecen metas planetarias para contrarrestar los efectos del cambio climático. Al considerar la dispersión espacial e intergeneracional de los daños, el principio establece como exigencia de justicia que los generadores del cambio climático deben responder por él ${ }^{23}$. Asumiendo la premisa igualitaria sobre el derecho de cada cual a un uso igual del recurso atmosférico, es posible argumentar del siguiente modo en el caso de los refugiados climáticos: todos tienen un derecho igual a utilizar y eventualmente cargar el medioambiente atmosférico en tanto este uso no implique la violación de derechos fundamentales (es decir: el límite al uso igual estaría delimitado por las capacidad restringida de absorción de nuestro planeta en relación a su capacidad para mantener un medioambiente que no implique la violación de derechos humanos básicos). Todos aquellos que utilicen más que su parte violan el derecho igual de los otros. Por consiguiente, deben compensarlos en proporción a lo extra utilizado. En el caso de los refugiados climáticos esto sucede asumiendo los costos relacionados con la movilidad en proporción a lo extra utilizado. En la próxima sección determinaré las exigencias de este principio con respecto a los refugiados climáticos con mayor precisión.

Un eje central de este argumento refiere a la determinación de los agentes: ¿se trata de individuos o de Estados? En mi opinión, la respuesta más evidente es estatista ${ }^{24}$. Por lo demás, así se suele considerar en los debates internacionales en torno a la protección medioambiental. Por tanto las imputaciones de responsabilidad no aplican a individuos, sino que a Estados (dependiendo de las definiciones, naciones o pueblos). Hay buenas razones para esta estrategia argumentativa. Ella asume la organización internacional como un contexto dado y de este modo torna más plausible la implementación de políticas medioambientales (los Estados aceptan o rechazan normas vinculantes del derecho internacional, y los gobiernos de los Estados establecen e implementan políticas medioambientales). Además, hace posible el funcionamiento del orden internacional en tanto los Estados (aunque no en todos los casos) ocupan espacios temporales más extendidos que los que ocupan individuos, haciendo posible las imputaciones de responsabilidad en un espacio temporal más extendido.

${ }^{23}$ Compare GARDINER, Stephen. Ethics and Global Climate Change; IDEM. The Global Warming Tragedy and the Dangerous Illusion of the Kyoto Protocol.

${ }^{24}$ Aceptar la premisa estatista en la determinación de los agentes no implica una distribución igualitaria entre Estados del recurso atmosférico. ¿Cómo se calcula el derecho de cada Estado a utilizar la atmosfera? La respuesta más sensible debe relacionarse con el derecho igual de los individuos explicitado en el punto anterior: es la suma de individuos en cada Estado la que determina el uso del recurso atmosférico que legítimamente puede realizar cada Estado. En todo caso, al disociar a los Estados en cuanto agentes de sus demandas legítimas (que refieren al número de individuos bajo su poder) surgen incentivos perversos. Por ejemplo: aumentar la población para aumentar así el nivel del uso legítimo del medioambiente. 
Y tercero: en un mundo organizado de acuerdo al paradigma estatista, son los Estados los que pueden hacer a los individuos responsables de sus actos.

Este principio está sujeto a una serie de críticas atingentes. Sólo mencionaré dos de ellas: (1) El problema del conocimiento ${ }^{25}$ : ¿̇somos responsable de nuestros actos cuando no sabemos - o si asumimos una obligación epistémica - cuando no podemos saber acerca de sus consecuencias? La respuesta parece ser que no. Si es así, entonces este principio aplica sólo a partir del momento en que los Estados pueden saber acerca de los efectos del sobreuso del recurso atmosférico. (2) El problema de la causalidad no lineal: el principio discutido refiere a una estructura simple. Si un agente $X$ con su acción amenaza y hace peligrar a $Y$, al menos a partir del momento en que $X$ o $Y$ lo saben, debe acabar con su acción y ayudar a subsanar los daños producidos a Y. El problema es que esta estructura no se da siempre. Este es, por ejemplo, el caso de Noruega ${ }^{26}$. Noruega obtiene su energía principalmente de represas que no contaminan el medioambiente atmosférico. Por lo tanto, de acuerdo al principio discutido no debiese hacerse cargo de los costos, al menos de los relacionados con aquellos daños producidos hoy. Sin embargo, Noruega es un gran exportador de petróleo que se utiliza en actividades altamente contaminantes. Estas exportaciones llevan a que Noruega tenga una alta calidad de vida. ¿No debería Noruega ser responsable de una parte de estos daños y costos relacionados? Aquí quedan brechas importantes sin cubrir al asignar responsabilidad.

La tercera respuesta es que los beneficiados de las acciones que dañan el medioambiente atmosférico, ya sean acciones del pasado o del presente, deben hacerse cargo de los $\operatorname{costos}^{27}$ de mitigación, adaptación, aceptación y compensación. En contraste a la respuesta anterior, en este caso el agente responsable no es el que pone la causalidad en movimiento, sino el que se beneficia de su ocurrencia.

La justificación de este principio puede apelar a consideraciones igualitarias que se expresarían al considerar la condición de free rider y de parásito. Un free rider es aquel que se beneficia de la acción de otro sin asumir los costos. Un parásito es aquel que obtiene beneficios, pero desvía los costos a un tercero. Así, un propietario de barco que obtiene beneficios del faro, sin hacerse cargo de sus costos, es un free rider. Mientras que el dueño de industria que contamina la atmósfera sin compensar es un parásito ${ }^{28}$. Por ejemplo, si la generación pasada en EEUU llevó a cabo un proceso de industrialización que causó daños a un tercero, por ejemplo acelerando el cambio climático y así inundando Bangladesh (que no se benefició de este proceso), entonces esa generación de estadounidenses es

\footnotetext{
${ }^{25}$ CANEY, op. cit.

26 GESANG, Bernward. Klimaethik.

27 Compare GOSSERIES, Axel. Historical Emissions and Free-Riding.

${ }^{28}$ GAUTHIER, David. Morals by Agreement.
} 
parásito de Bangladesh. Por su parte, si esa generación desaparece y su lugar es ocupado por una nueva generación que se beneficia del proceso de desarrollo de la generación anterior sin emitir ella misma gases contaminantes, entonces esa nueva generación es free rider de Bangladesh, porque no se está haciendo cargo de los costos pero si disfrutando los beneficios ${ }^{29}$. A pesar de sus múltiples dificultades interpretativas, este principio ofrece algunas ventajas:

No se presenta el problema de la causalidad no lineal: aunque Noruega no contamine, dado que su riqueza se basa en la exportación de productos contaminantes, se beneficia de la destrucción del medioambiente atmosférico y, por consiguiente, debe hacerse cargo de los costos en proporción a su beneficio ${ }^{30}$.

Soluciona el desafío que las generaciones contaminadoras pasadas plantean al principio anterior: puede que estas generaciones ya no existan, pero eso no remueve ni en un ápice la responsabilidad con respecto a los costos de todos aquellos que se beneficien de sus acciones contaminantes. Así "en lo referente a las emisiones históricas el pueblo americano es un free rider de Bangladesh, y en lo referente a las emisiones actuales es un parásito" ${ }^{\prime 31}$.

Una crítica a este principio refiere a su carácter paradójico. Si X goza de un alto bienestar debido a la sobreutilización de recursos atmosféricos por parte de $Y$, de modo que debe asumir los costos, esto puede llevar a que algunos beneficiados deban cargar con costos que impliquen la perdida de todos sus beneficios, llegando incluso a encontrase en situación de absoluta pobreza. Por tanto, es razonable preguntar dónde termina la responsabilidad por los costos. Si no se define algún mínimo (lo que implica aceptar otro principio), el principio

${ }^{29}$ Un problema con este tipo de argumentos es que deben necesariamente recurrir a consideraciones morales acerca del estatus del free rider. Es decir, ser free rider implicaría no cumplir con la propia responsabilidad u obligaciones. Pero esto no debe ser necesariamente así (NOZICK, Robert. Anarchy, State and Utopia). No toda acción de free rider es moralmente criticable. Por ejemplo, si me beneficio de la seguridad de mi nuevo vecino, que casualmente es el presidente, no por ello debería hacerme cargo de los costos. Y si no lo hago es implausible afirmar que no estoy cumpliendo con mis obligaciones o responsabilidad. Este tipo de argumentos evidencia que ser un free rider no es sinónimo de no cumplir las obligaciones debidas.

${ }^{30}$ GESANG, op. cit.

${ }^{31}$ GOSSERIES, op. cit., p. 45. Considerándolo de un modo aislado, este principio está sujeto a una crítica fuerte: El problema de la irreductibilidad de beneficios a emisiones. Es decir, el beneficio del que disfrutan algunos agentes (mayor disposición de ciertos bienes estipulados de acuerdo a alguna teoría), no se puede reducir a emisiones. Muchos otros elementos entran en la ecuación. Entre otros, creatividad, trabajo duro, inversiones a largo plazo, etc. Retrotraer el beneficio exclusivamente a las emisiones es una restricción injustificada. Contra esta crítica se suele argumentar que los procesos de industrialización han estado acompañados de consumo masivo de energías contaminantes de la atmosfera. Sin embargo, aunque esto sea así, no todos los procesos de desarrollo económico, al menos a partir de algún momento, han sido igualmente contaminantes. Así se mantiene que el uso de recursos atmosféricos es uno más entre otros factores que han producido los beneficios actuales. Si esto es así, entonces hay que restringir este principio a la proporción de los beneficios que refieren al sobreuso del recurso atmosférico. 
es paradójico en el sentido que la responsabilidad por el beneficio disfrutado puede hacer perder no sólo el beneficio, por el que ciertamente no éramos responsable, sino que puede llevar a algunos individuos a pertenecer al grupo de los más desposeídos, sin poder ser considerados responsables por esto. Es decir, la mala suerte de tener una herencia corrupta.

En mi opinión, partiendo desde un punto de partida igualitario definido en relación al uso igual del recurso atmosférico, la asignación de costos por los refugiados climáticos debe realizarse atendiendo al trabajo en conjunto de los dos últimos principios. Debido a su carácter histórico, el principio "el que contamina, paga", sujeto a las limitaciones de conocimiento, hace cargar a los Estados los costos de sus propias acciones. Por otra parte, el principio "el que se beneficia, paga" permite incluir en la matriz de asignación de costos a los free riders. Así, cada Estado es responsable de los costos, ya sean de mitigación, de adaptación, así como de compensación y recepción de refugiados climáticos, en proporción al sobreuso del recurso atmosférico, y al beneficio obtenido, aunque este beneficio no sea reducible a acciones causadas por ellos mismos. En cualquier caso, la aplicación de estos principios debe ser limitada por un principio estipulativo de algún mínimo (y así evitar los resultados paradójicos mencionados).

Este principio de asignación de costos debe ser aun determinado. Como vimos, los costos pueden ser de diferentes tipos. Una pregunta central es si se pueden realizar trade-offs entre ellos.

\section{Trade-offs y responsabilidad por los refugiados climáticos}

A continuación, consideraré cuatro preguntas relativas a la aplicación del principio elaborado para asignar los costos del desplazamiento climático:

Primero: ¿Son legítimos los trade-offs entre los diferentes tipos de costos o ellos deben ser distribuidos en proporción igual en cada Estado? Por ejemplo, ¿puede un Estado asumir más costos de mitigación, adaptación y compensación en razón de aceptar menos refugiados climáticos? A mi juicio, un aporte a los costos de mitigación, adaptación y compensación que supere lo que la responsabilidad indica, no debe disminuir la responsabilidad relacionada a la aceptación de refugiados. Por una parte, este proceso de asignación implicaría cálculos complejos - aunque no por ello irrealizables - (la relación entre, por ejemplo, aceptar refugiados climáticos y hacerse cargo de mayores costos de mitigación para así no aceptarlosi - cuántos metros de dique vale cada refugiado?). Por otra parte, y más importante, no debe ser posible comprar el derecho a violar derechos. La justicia correctiva en que se basa el principio elaborado aspira a restituir (aunque sea imposible y toda restitución sea aproximativa) una situación 
original ilegítimamente violada, y no puede, por tanto ser utilizada como un mecanismo para legítimamente realizar estas violaciones. En este sentido, si bien los costos de mitigación, adaptación y compensación admitirían trade-offs entre ellos, la obligación (y los costos relacionados con el cumplimiento de esta obligación) de aceptar refugiados climáticos gozaría de una cierta prioridad.

Segundo: ¿Puede un Estado cumplir su obligación de aceptación mediante un tercer Estado que esté dispuesto a aceptarlos - por ejemplo, debido a pagos o condonación de deudas por parte del Estado responsable? Este proceso daría lugar a una especie de mercado de certificados de desplazados: los Estados podrían adquirir el derecho a contaminar (y así a participar en el cambio climático) mediante un certificado de aceptación de inmigrantes que luego podrían vender a un tercer Estado. En mi opinión, este proceso banaliza la situación y la injusticia sufrida por los refugiados. El derecho de los refugiados climáticos no puede ser considerados de un modo análogo a certificados de emisiones de carbono transables en el mercado. Vale el punto anterior: la obligación de aceptar refugiados climáticos es prioritaria.

Tercero: ¿Si no hay refugiados climáticos que quieran migrar a un país particular, debe el Estado correspondiente asumir otro tipo de costos o debe obligarse a los desplazados a encontrar refugio en ese país? En mi opinión, estos Estados deben hacerse cargo de otro tipo de costos. Después de todo, son los desplazados los dañados por la acción de estos Estados y, por tanto, aquellos cuya voluntad debe tener prioridad al momento de obtener refugio. Sin embargo, este proceso puede producir incentivos perversos: asumiendo que cumplir la obligación de aceptación implica costos más altos (ya que no son sólo monetarios), todos aquellos países que no quieran aceptar refugiados (en razón, por ejemplo, de entendimientos culturales o raciales) estarían motivados para tornarse en países especialmente poco atractivos para los refugiados, haciendo uso del instrumentario legal para tornar la situación de los refugiados en una de mayor indefensión, y aprovechando y fomentando procesos sociales de rechazo a los refugiados. Por una parte, esto reflejaría un deseo de no hacerse cargo de los costos que se retrotraen a la propia responsabilidad vía justicia correctiva. Por otra parte, este modus operandi impondría en otros Estados la obligación de aceptar a los refugiados que, por buenas razones, no quieren serlo en esos países. Una solución sería hacer a esos Estados responsables por los otros tipos de costo pero con una tasa de convertibilidad tan elevada entre el costo de aceptación y los otros tres tipos de costo, que todos los países se vean incentivados a transformarse en países de aceptación escogidos por los refugiados climáticos.

Cuarto: ¿Qué sucede con los desplazados internos, es decir, que no requieren ser recibidos por otros Estados? ¿Y qué sucede en el caso de aquellos 
desplazados hacia países que no son ellos mismos responsables por los costos discutidos? La primera pregunta es relevante, porque la mayor cantidad de desplazados climáticos son internos. Y en muchos casos esos países no son responsables por los costos. En mi opinión, en estos casos los Estados responsables están obligados a asumir los costos en relación a los Estados en que se desplazan los refugiados. Ciertamente, esto produce una serie de incentivos perversos en los países en que se produce el desplazamiento interno que alimentan la corrupción. La segunda pregunta es también importante, porque en muchos casos son los países vecinos los que reciben a los refugiados, y en muchos casos ellos son tan poco responsables como los países de origen por los desplazados climáticos. En este caso debiese valer lo mismo que en el anterior: los Estados responsables están obligados a asumir los costos en relación a los Estados en que se desplazan los refugiados.

La asignación de costos en relación a los refugiados climáticos es un tema complejo. No he pretendido tratarlo a cabalidad, ni dar respuesta a todas las interrogantes que surgen, sino tan sólo diseñar una línea normativa general para guiar nuestras nuestros juicios.

\section{Bibliografía}

ANDERSON, Elizabeth. How should egalitarians cope with market risks? Theoretical Inquiries in Law, v. 9, n. 1, 2008.

. What is the point of equality. Ethics, v. 109, n. 2, 1999.

ARNESON, Richard. Luck egalitarianism and prioritarianism. Ethics, v. 110, n. 2, 2000.

BELL, Derek R. Environmental refugees: what rights? which duties? Res Publica, v. 10, 2004, p. 135-152.

BLACK, Richard. Environmental refugees: myth or reality? 2001. Disponible en: $<$ http://www.unhcr.ch/refworld/pubs/pubon.htm>.

BROWN, Lester R. Environmental refugees. 2002. Disponible en: $<$ http://www.foes. org.au/ci/ci_ecoref.htm>.

BROWN, Oli. Migration and Climate Change. IOM, n. 31, 2008.

CANEY, Simon. Cosmopolitan Justice, Responsibility, and Global Climate Change. Leiden Journal of International Law, v. 18, 2005.

COHEN, Gerald A. On the currency of egalitarian justice. Ethics, v. 99, n. 4, 1989.

DWORKIN, Ronald. Equality, luck, and hierarchy. Philosophy and public affairs, v. 31, n. 2, 2003.

EL-HINNAWI. Environmental Refugees. Nairobi: United Nations Environmental Programme, 1985.

FREEMAN, Samuel. Justice and the social contract. New York: OUP, 2006.

GARDINER, Stephen. Ethics and Global Climate Change. Ethics, v. 114, 2004.

. The Global Warming Tragedy and the Dangerous Illusion of the Kyoto 
Protocol. Ethics and International Affairs, v. 18, 2004. . A Perfect Moral Strom. Oxford: Oxford University Press, 2011.

GAUTHIER, David. Morals by Agreement. Oxford: Oxford University Press, 1986. GESANG, Bernward. Klimaethik. Berlin: Suhrkamp, 2011.

GOSSERIES, Axel. Historical Emissions and Free-Riding. Ethical Perspectives, v. 11, n. 1, 2004.

GRAY, Clark. Environmental refugees or economic migrants? Population Reference Bureau, 2010. Disponible en: <http://www.prb.org/Publications/Articles/2010/ environmentalmigrants.aspx $>$.

GRUBB, Michael. Seeking fair weather: ethics and the international debate on climate change. International Affairs, v. 71, n. 3, 1995.

KANT, Immanuel. La Metafísica de las Costumbres [1797]. Madrid: Tecnos, 1989. . Hacia la Paz Perpetua [1795]. Madrid: Biblioteca Nueva, 1999.

LOEWE, Daniel. Inmigración y el Derecho de Gentes de John Rawls. Revista de ciencia política, v. 27, n. 2, 2007.

. Los náufragos de nuestro mundo. El caso de los refugiados. Arbor, v. 186, n. 744, 2010.

MILLER, David. Equality and justice. In MASON, Andrew (ed.). Ideals of equality. Oxford: Blackwell, 1998.

MINURA, Nobuo et alii. Small Island. In PARRY, Martin; CANZIANI, Osvaldo; PALUTIKOF, Jean; Van der LINDER, Paul; HANSON, Clair (eds.). Impacts, adaptation and vulnerability. Contribution of the Working Group III to the Fourth Assessment Report of the Intergovernmental Panel on Climate Change. Cambridge: Cambridge University Press, 2007, p. 687-716.

MORTREUX, Colette; BARNETT, Jon. Climate change, migration and adaptation in Funafuti, Tuvalu. Global Environmental Change, v. 19, p. 105-112, 2009.

MYERS, Norman. Environmental Refugees: An emergent security issue. $13^{\text {th }}$ Economic Forum, 2005. Disponibleen: < http://www.osce.org/eea/14851 ?download=true >. NOZICK, Robert. Anarchy, State and Utopia. Basic Books, 1974.

NUSSBAUM, Martha C. Frontiers of Justice. Cambridge: Harvard University Press, 2006. . Women and Human Development. Cambridge: Cambridge University Press, 2000.

RAWLS, John. Justice as Fairness (KELLY, Erin, ed.). Cambridge, MA: Harvard University Press, 2001.

SCHEFFLER, Samuel. Equality and tradition. New York: Oxford University Press, 2010. . What is egalitarianism? Philosophy and public affairs, v. 31, n. 1, 2003.

SEGALL, Shlomi. Equality and opportunity. Oxford: Oxford University Press, 2013. SEN, Amartya. Development as Freedom. Nueva York: Anchor Books, 1999.

. Equality of What. In MCMURRIN, Sterling (ed.). Tanner Lectures on Human Values. Cambridge: Cambridge University Press, 1980. 
. Inequality Reexamined. New York: Russell Sage Foundation, Harvard University Press, ([1992]1997).

SHUE, Henry. Avoidable Necessity: Global Warming, International Fairness, and Alternative Energy. In SHAPIRO, lan; DeCEW, Judith Wagner (eds.). NOMOS XXXVII: Theory and Practice. New York: New York University Press, 1995.

. The Unavoidability of Justice. In HURRELL, Andrey; KINGSBURY, Benedict (eds.). The International Politics of the Environment. Oxford: Oxford University Press, 1992.

SINGER, Peter. Famine, Affluence, and Morality. Philosophy and Public Affairs, v. 1, n. 1, 1972.

. One World: The Ethics of Globalization. New Haven and London: Yale University Press, 2004.

. The Life You Can Save. New York: Random House Publishing Group, 2009.

SMITH, Mick. An ethics of place: radical ecology, postmodernity and social theory. Albany: SUNY Press, 2001.

STERN, Nicholas (ed.). The Economics of Climate Change: The Stern Review. Cambridge: Cambridge University Press, 2006.

TAN, Kok-Chor. Justice, institutions, and luck. Oxford: Oxford University Press, 2012.

\section{Abstract}

\section{Climate refugees: who should assume the costs?}

This paper argues for the concept of "Climate refugees". It proposes that the responsibility for their displacement and the associated costs, including their reception by other states, should be allocated among states according tot heir corresponding use of atmospheric resources and their benefits obtained by using those resources.

Keywords: climate change, forced displacement, climate refugee, cost allocation principles.

Recebido para publicación en 18/08/2014.

Aceptado para publicación en 03/11/2014.

Received for publication in August, 18 ${ }^{\text {th }}, 2014$. Accepted for publication in November, 03 ${ }^{\text {th }}, 2014$. 